\title{
On the Exact Distribution of Mutual Information of Two-User MIMO MAC Based on Quotient Distribution of Wishart Matrices
}

\author{
Gabriel Pivaro $^{1 *}$, Santosh Kumar ${ }^{2}$ and Gustavo Fraidenraich ${ }^{1}$ \\ The pre-print version of this work can be found in https://arxiv.org/pdf/1601.03439v1.pdf.
}

\begin{abstract}
We propose an exact calculation of the probability density function (PDF) and cumulative distribution function (CDF) of mutual information (MI) for a two-user multiple-input multiple-output (MIMO) multiple access channel (MAC) network over block Rayleigh fading channels. This scenario can be found in the uplink channel of MIMO non-orthogonal multiple access (NOMA) system, a promising multiple access technique for $5 \mathrm{G}$ networks. So far, the PDF and CDF have been numerically evaluated since MI depends on the quotient of two Wishart matrices, and no closed form for this quotient was available. We derive exact results for the PDF and CDF of extreme (the smallest/the largest) eigenvalues. Based on the results of quotient ensemble, the exact calculation for PDF and CDF of mutual information is presented via Laplace transform approach and by direct integration of joint PDF of quotient ensemble's eigenvalues. Furthermore, our derivations also provide the parameters to apply the Gaussian approximation method, which is comparatively easier to implement. We show that approximation matches the exact results remarkably well for outage probability, i.e., CDF, above 10\%. However, the approximation could also be used for $1 \%$ outage probability with a relatively small error. We apply the derived expressions to investigate the effects of adding antennas in the receiver and its ability to decode the weak user signal. By supposing no channel knowledge at transmitters and successive decoding at receiver, the capacity of the weak user increases and its outage probability decreases with the increment of extra antennas at the receiver end.
\end{abstract}

Keywords: Multiple access channel, Multiple-input multiple-output, Non-orthogonal multiple access, Mutual information, Outage probability, Rayleigh fading, Wishart matrices, Quotient ensemble, Extreme eigenvalues, Gap probabilities

\section{Introduction}

It is now well acknowledged that the use of multiple-input multiple-output (MIMO) scheme is crucial to increase the capacity and reliability of wireless systems. MIMO setup provides several benefits such as higher received power via beamforming, higher channel capacity via spatial multiplexing without increasing bandwidth or transmission power, and improved transmission robustness via diversity coding [1]. Current cellular systems such as 4G Long-Term Evolution (LTE) are using MIMO, and the next

*Correspondence: gfp.1@hotmail.com

'Department of Communications, State University of Campinas (Unicamp), Campinas, SP, Brazil

Full list of author information is available at the end of the article generation system such as $5 \mathrm{G}$ considers the deployment terminal with dozens of antennas, the so-called massive MIMO. A new scheme called non-orthogonal multiple access (NOMA) has been considered as a potential solution to improve the system capacity of future wireless systems due to its superior spectral efficiency $[2,3]$. The basic principle of NOMA is to serve multiple users by power domain multiplexing at transmitter and successive interference cancellation (SIC) at receiver, which can achieve the capacity region of the downlink addictive white Gaussian noise channel and significantly outperform the orthogonal multiple access (OMA) schemes [3].

In this paper, we derive exact expressions to obtain the distribution and the outage probability of the mutual 
information for a two-user MIMO multiple-access channel (MAC). To the best of our knowledge, no exact expressions were derived before, under the following assumptions: channel state information at receiver (CSIR), block Rayleigh fading channel, and successive decoding [4]. The main difficulty to derive exact expressions for this scenario is that the mutual information is a random variable that depends on the quotient of two Wishart matrices [5]. The recent exact analysis of quotient ensemble involving Wishart matrices led to the availability of the corresponding joint probability density function (JPDF) of the eigenvalues [6]. This opened the possibility to describe, in an exact manner, the behavior of the mutual information in a scenario such as MIMO NOMA uplink. Therefore, we derive the exact expressions related to the mutual information that allows, for example, the analysis of the impact of adding more antennas at the base station on the performance of network. In addition, we also present, for the first time in literature, the probability distributions and densities of extreme eigenvalues of the quotient ensemble.

We emphasize that the scenario proposed here is of practical interest since when there is no channel state information at the transmitter (CSIT), then the transmitter encodes its messages with a fixed rate (fixed power) $[1,7]$. However, under the slow-fading scenario with Rayleigh distribution, the signal transmitted could not be properly decoded at the receiver. In this case, an outage event occurs [1]. Our aim here, is to track the outage probability based on the message rate, on the number of antennas at all nodes, and the signal power. The proposed two-user scenario appears in existing studies for the implementation of NOMA, which has been suggested as a possible solution to pair two users whose channel conditions are very different [8]. We assume a SIC scenario [4], where the first user to be decoded is affected for the signal of the second user (that experiences an interference-free scenario), and therefore called weak user in NOMA notation. Here, we focus on the mutual information distribution of the weak user. With our results, we can quantify the performance improvement achieved with extra power or antennas.

\subsection{Related works}

The possible application of MIMO in wireless systems probably gained much more attention after Telatar's canonical work [9]. Telatar has shown that the capacity of a MIMO system is directly related to the realizations of the random channel matrix. These realizations are characterized by the probability density function (PDF). However, since the matrix dimensions grow as the number of antennas in the system increases, evaluation of the capacity is not a straightforward task. One key contribution of Telatar's work was to use random matrix theory
(RMT) to show that instead of working with the matrix PDF, the distribution of the mutual information could be accessed just by using the JPDF of the matrix eigenvalues. This is possible because of the invariant nature of the mutual information expression under unitary conjugation. Wang and Giannakis [10] showed that the mutual information could be well approximated by a Gaussian distribution, therefore, generating a Gaussian approximation (GA). Since a Gaussian distribution is fully characterized by its mean and variance, the problem reduces to working out these two parameters. The calculation of mean of mutual information yields the ergodic capacity, while the Gaussian approximation of mutual information can be used to obtain the outage probability.

The case of a single-user MIMO channel has been extensively studied. In [11], a closed-form expression for ergodic capacity was derived for any number of transmit and receive antennas for Rayleigh fading. The exact distribution of mutual information was presented in [12] for dual MIMO systems under Rician fading. In [13], the MIMO channel capacity over the Hoyt fading channel was presented. In [14], a random matrix model for the Nakagami- $q$ (Hoyt) fading MIMO communication channels with arbitrary number of transmitting and receiving antennas is considered. The Gaussian approximation was investigated in [15] for the Rician fading channel in the asymptotic regime of large number of transmitting and receiving antennas. In [16], the authors showed that Gaussian approximation remains quite robust even for large signal-to-noise ratio (SNR) for the case of unequal numbers of transmitting and receiving antenna arrays, while it deviates strongly from the exact result for equal number of antenna arrays. Beside the single-user scenario, MIMO systems have been studied in a variety of multi-user networks such as Broadcast Channel (BC), Interference Channel (IC), MAC, and Relay Channel. Earlier, much effort was devoted to extend the already known results for single-antenna case to the MIMO case [7]. Recent works are investigating how multiple antennas can be utilized to reduce interference in multi-user scenarios [17].

Although much investigation has been conducted to determine the capacity region and ergodic capacity of MIMO MAC network, only a few works have focused on the determination of outage probability for this channel. The authors in [17], assuming correlated Rayleigh fading in a multiuser MIMO beamforming network with channel distribution information (CDI), derived a closed-form expression for the outage probability. This expression was used to create algorithms for joint transmit/receive beamforming and power control to minimize the weighted sum power in the network while guaranteeing this outage probability. In [18], the authors derived closed-form expressions for outage probability in MIMO IC under the assumption of Gaussian-distributed CSI error and 
derived the asymptotic behavior of the outage probability as a function of several system parameters based on the Chernoff bound. In [19], the authors compared the performance in terms of capacity and maximum throughput, of a BC multiuser MIMO system and a MIMO timedivision multiple-access (TDMA) MIMO system. Their key assumption is that the number of transmit antennas is much larger than the number of receive antennas at each user and complete knowledge of the CSIT. In [20], the authors analyzed asymptotic weighted sum rate maximization in the MIMO MAC. In [21], the authors propose an iterative algorithm to design optimal linear transmitters and receivers in a $K$-user frequency-flat MIMO IC with channel state information at the transmitter and receiver (CSITR).

Recently, many efforts have been conducted to investigate the application of MIMO techniques to NOMA systems, as a possibility to enhance the performance gains of NOMA. In [22], a novel MIMO-NOMA framework for downlink and uplink transmission is proposed by applying the concept of signal alignment. Closed-form analytical results are developed to facilitate the performance evaluation of the proposed framework. The impact of fixed power allocation and cognitive radio inspired power allocation on the performance of MIMO-NOMA is also investigated. In [3], the ergodic capacity maximization problem is first studied for the Rayleigh fading MIMO-NOMA systems with statistical CSIT. The authors propose both optimal and low complexity suboptimal power allocation schemes to maximize the ergodic capacity of MIMO-NOMA system with total transmit power constraint and minimum rate constraint of the weak user. Numerical results show that the proposed NOMA schemes significantly outperform the traditional OMA scheme. A new design of precoding and detection matrices for MIMO-NOMA is proposed, in [23], and its performance is analyzed for the case with a fixed set of power allocation coefficients. Computer simulation results are provided to facilitate the performance evaluation of MIMO-NOMA and also demonstrate the accuracy of the developed analytical results. A Pseudo Double Scattering Channel (PDSC) Matrix assumption is proposed, in [24], for the downlink NOMA within the massive MIMO systems. Afterwards, the CDF and the outage probability of such a system are investigated with the aid of random matrix and statistics theories. The derivations obtained are verified through numerical simulations. The authors find out that with the increase of the number of antennas, the outage probability is reduced. In [25], for singleantenna case, the authors analyze the outage capacities of SIC and Joint Decoding (JD) in the case of single-block transmission over a two-user Gaussian MAC with partial CSIT. Results show that JD can achieve a sum-rate gain of up to $10 \%$ or sum-power gain of $0.8 \mathrm{~dB}$.

\subsection{On the paper contributions}

The key contributions of this paper are to obtain exact results for the cumulative distribution function (CDF) and PDF of (i) the extreme eigenvalues of the quotient ensemble comprising two Wishart matrices and (ii) mutual information for the case when it is a random variable and again depends on the quotient of two Wishart matrices. As we show in Section 2, both the PDF and CDF of mutual information could be written as a function of JPDF, as in the single-user case. We invoke the closed form of JPDF of eigenvalues for the quotient ensemble derived in [6] in Section 3. In Section 4, we derive, for the first time in literature, closed-form expressions of CDF and PDF for the extreme eigenvalues that can be further explored to calculate the outage probability in beamforming scenario [24]. With the aid of JPDF, we propose two different methods in Section 5 to derive the exact expressions for PDF and $\mathrm{CDF}$ of the mutual information. The first one relies on direct integration of the JPDF, while the second one is to use Laplace transform approach.

Although the exact expressions for PDF and CDF of the mutual information involve integrals, they provide analytical exact results. Besides the two exact solutions indicated above, we also present in Section 5 the means to obtain the mean and variance of mutual information using two approaches. The first one is based on employing the Laplace transform of mutual information as momentgenerating function. The second one, on the other hand, uses the first- and the second-order marginal densities (one-point and two-point correlation functions, respectively) of the eigenvalues of the quotient ensemble. With these parameters, we also obtain the Gaussian approximation that is straightforward to use and matches the exact results.

Finally, we use the above derivations to analyze the outage probability for a two-user MIMO MAC in a low-SNR scenario. The numerical results show that increasing the number of antennas at the base station decreases the outage probability for the weak user signal. The results are evaluated in Section 6, where Monte Carlo simulations show perfect agreement with all our analytical expressions. We characterize the possible outage values where the Gaussian approximation matches the exact results extremely well.

\section{System model, mutual information probability, and density and outage probability}

In this section, we first describe the system model under consideration-the two-user MIMO MAC, a common network that usually appears in the uplink of a cellular-type system [7]. To understand how much information this two-user MIMO MAC could convey, we need to characterize its mutual information. Since the mutual information is a random variable that depends on realizations 
of the channel matrix, our goal here is to express the mutual information PDF as a function of channel matrices' eigenvalues, which reduces the complexity of the problem. Afterwards, we define the outage probability, that is the mutual information CDF and our main metric to analyze the performance of the two-user MIMO MAC. These expressions are the starting point to derive the exact results proposed in this work.

\subsection{System model}

Consider the two-user MIMO MAC network depicted in Fig. 1. The base station (BS) has $n$ receiving antennas and each of the users' equipment or mobile stations has $n_{i}$, $i=A, B$, transmitting antennas. We assume that $n_{i} \geq n$, following the same assumption made in [8], where $n_{i} \geq$ $n / 2$.

The users transmit $\mathbf{u}_{i} \in \mathbb{C}^{n_{i} \times 1}$, that is circularly symmetric complex Gaussian vector with zero-mean and positive definite covariance matrices $\mathbf{Q}_{i}$. Users are subject to an individual power constraint of $\operatorname{tr}\left(\mathbf{Q}_{i}\right) \leq n_{i}$, where $\operatorname{tr}(\cdot)$ is the trace of a matrix. Let $\mathbf{v} \in \mathbb{C}^{n \times 1}$ denote the received signal at BS. The $\mathbf{w} \in \mathbb{C}^{n \times 1}$ is the noise vector circularly symmetric complex Gaussian with zero-mean and covariance matrix $\mathbf{I}_{n}$, where $\mathbf{I}_{n}$ is the $n \times n$ identity matrix. The $n \times n_{i}$ dimensional channel matrix is denoted by $\mathbf{H}_{i}$, and its entries are independent and identically distributed (i.i.d.) complex Gaussian random variables with zero-mean and unit variance. Therefore, $\mathbf{H}_{i}$ is governed by the probability density

$$
p_{H}\left(\mathbf{H}_{i}\right) \propto e^{-\operatorname{trH}_{i} \mathbf{H}_{i}^{\dagger}} .
$$

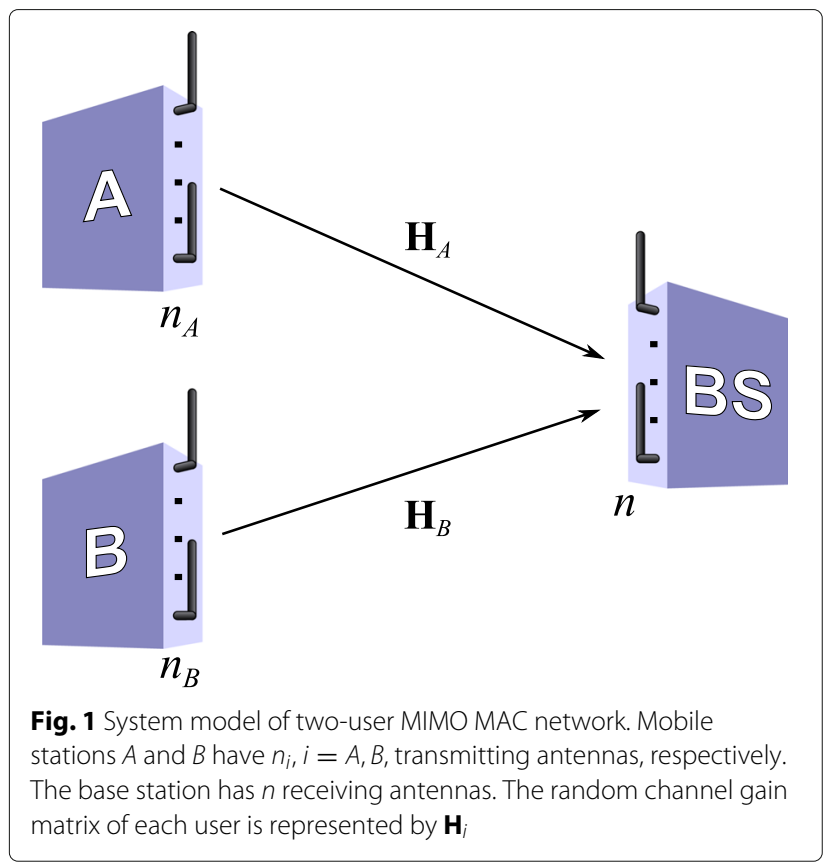

Here, $\dagger$ denotes the conjugate transpose. The received signal at $\mathrm{BS}$ is given by

$$
\mathbf{v}=\sqrt{a} \mathbf{H}_{A} \mathbf{u}_{A}+\sqrt{b} \mathbf{H}_{B} \mathbf{u}_{B}+\mathbf{w}
$$

where " $a=\mathrm{SNR}_{A} / n_{A}$ and $b=\mathrm{SNR}_{B} / n_{B}$, and $\mathrm{SNR}_{i}$ are the normalized power ratios of $\mathbf{u}_{i}$ to the noise (after fading) at each receiver antenna of BS" as stated in [26].

\subsection{Mutual information}

The BS wishes to recover $\mathbf{u}_{i}$ from $\mathbf{v}$. Since $\mathbf{u}_{i}$ and $\mathbf{v}$ are random variables, we use the mutual information to measure how much information BS is able to recover. Then, the MIMO MAC capacity region, assuming SIC decoding, is given in terms of mutual information of $A$ and $B$ as $[3,4,7]$

$$
\begin{aligned}
\mathcal{I}_{A} & =\log _{2}\left[\operatorname{det}\left(\mathbf{I}_{n}+\mathbf{A}+\mathbf{B}\right)\right]-\mathcal{I}_{B} \\
& =\log _{2}\left[\operatorname{det}\left(\mathbf{I}_{n}+\left(\mathbf{I}_{n}+\mathbf{B}\right)^{-1} \mathbf{A}\right)\right],
\end{aligned}
$$

and

$$
\mathcal{I}_{B}=\log _{2}\left[\operatorname{det}\left(\mathbf{I}_{n}+\mathbf{B}\right)\right],
$$

where $\mathbf{A}=a \mathbf{H}_{A} \mathbf{H}_{A}^{\dagger}, \mathbf{B}=b \mathbf{H}_{B} \mathbf{H}_{B}^{\dagger}$, and $\operatorname{det}(\cdot)$ is the determinant of a square matrix. We note that the positive definite matrices $\mathbf{A}$ and $\mathbf{B}$ are from Wishart distributions:

$$
\begin{aligned}
& p_{A}(\mathbf{A}) \propto \operatorname{det}(\mathbf{A})^{n_{A}-n} e^{-a^{-1} \operatorname{tr} \mathbf{A}}, \\
& p_{B}(\mathbf{B}) \propto \operatorname{det}(\mathbf{B})^{n_{B}-n} e^{-b^{-1} \operatorname{tr} \mathbf{B}} .
\end{aligned}
$$

As determinant is invariant under unitary transformation, applying a procedure similar to that in [9], we can rewrite (3) as function of the eigenvalues $\lambda_{j}, j=1, \ldots, n$ of the $n \times n$ complex matrix $\mathbf{W}$ as

$$
\mathcal{I}_{A}=\log _{2} \operatorname{det}\left(\mathbf{I}_{n}+\mathbf{W}\right)=\sum_{j=1}^{n} \log _{2}\left(1+\lambda_{j}\right)
$$

where

$$
\mathbf{W}=\left(\mathbf{I}_{n}+\mathbf{B}\right)^{-1} \mathbf{A}=\left(\mathbf{I}_{n}+b \mathbf{H}_{B} \mathbf{H}_{B}^{\dagger}\right)^{-1}\left(a \mathbf{H}_{A} \mathbf{H}_{A}^{\dagger}\right) .
$$

Note that we have assumed, without loss of generality, that BS decodes $A$ 's signal first and then $B$ 's signal. In this case, the rate of $A$ is affected by the interference caused by $B$ 's signal, which does not happen with $B$ [4]. In this case, (4) is the mutual information of a single-user MIMO channel and is characterized in $[9,10]$. On the other side, the mutual information for the MIMO MAC sum-rate given by $\left(\mathcal{I}_{A}+\mathcal{I}_{B}\right)$ has its PDF and CDF approximated in [27], and its mean exactly characterized in [28].

Therefore, in this work, we focus on the distribution and outage of mutual information of user A given in (6), that is known as the weak user in MIMO NOMA system notation [3]. 


\subsection{Outage probability and outage rate}

Now, we characterize the outage probability and outage rate, the metrics we chose to analyze the performance of the MIMO MAC network.

We consider in this work, the slow-fading scenario. In slow fading, with no CSIT, the transmitter encodes $\mathbf{u}_{i}$ with a fixed rate $R$ bits/s/Hz. An outage event could happen when the channel gain is too low for $\mathbf{u}_{i}$ to be recovered [1] The probability of occurrence of an outage event is known as outage probability and is given by ([4], Eq. (5.54)):

$$
\begin{aligned}
p_{\text {out }}(R) & =\operatorname{Pr}\left\{\mathcal{I}_{A}<R\right\} \\
& =\operatorname{Pr}\left\{\log _{2}\left[\operatorname{det}\left(\mathbf{I}_{n}+\mathbf{W}\right)\right]<R\right\} .
\end{aligned}
$$

The outage rate is defined in [10] as the rate $R$ for which the outage probability is at the given level $\varepsilon$ :

$$
R_{\text {out }}=\arg _{R}\left[p_{\text {out }}(R)=\varepsilon\right] .
$$

In other words, the outage rate is the rate conveyed subject to outage probability equal to $\varepsilon$ [1].

Since working with $\mathbf{W}$ is not straightforward because the number of integrals is related with the number of W's entries, we adopt similar procedure from [9] and rewrite (8) as a function of the eigenvalues of $\mathbf{W}$. Then, the outage probability is given by

$$
\begin{aligned}
p_{\text {out }}(R) & =\operatorname{Pr}\left\{\left(\prod_{i=1}^{n}\left(1+\lambda_{j}\right)\right)<2^{R}\right\} \\
& \stackrel{(\mathrm{a})}{=} \int_{0}^{\infty} \cdots \int_{0}^{\infty} \Theta\left(2^{R}-\prod_{j=1}^{n}\left(1+\lambda_{j}\right)\right) P\left(\lambda_{1}, \ldots, \lambda_{n}\right) d \lambda_{1} \ldots d \lambda_{n} \\
& =\int_{0}^{\infty} \cdots \int_{0}^{\infty} \Theta\left(R-\sum_{j=1}^{n} \log _{2}\left(1+\lambda_{j}\right)\right) P\left(\lambda_{1}, \ldots, \lambda_{n}\right) d \lambda_{1} \ldots d \lambda_{n},
\end{aligned}
$$

where $\Theta(\cdot)$ represents the Heaviside-theta function, with $\Theta(x)=0$ for $x<0$ and $\Theta(x)=1$ for $x>0, x \in \mathbb{R}$. Note that (a) follows because the theta function ensures that contribution to the probability comes only from the region where $\prod_{i=1}^{n}\left(1+\lambda_{j}\right)<2^{R}$. Evidently, determining the outage probability amounts to calculating the CDF of the mutual information.

Finally, the PDF of mutual information is obtained by differentiating (10) and is given by

$$
\begin{aligned}
p\left(\mathcal{I}_{A}\right) & =\left.\frac{d p_{\text {out }}(x)}{d x}\right|_{x=\mathcal{I}_{A}}=\int_{0}^{\infty} \cdots \int_{0}^{\infty} \delta \\
& \times\left(\mathcal{I}_{A}-\sum_{j=1}^{n} \log _{2}\left(1+\lambda_{j}\right)\right) P\left(\lambda_{1}, \ldots, \lambda_{n}\right) d \lambda_{1} \cdots d \lambda_{n},
\end{aligned}
$$

where $\delta(\cdot)$ is the Dirac-delta function ([29], pg. 1029).

The expressions (10) and (11) are the formal solutions to the outage probability and the density of mutual information. In Section 4, we plug the JPDF in (10) and (11) and present the final expressions. We also present an alternative form based on Laplace transform which is also exact and represent an alternative in terms of computation time. Finally, a Gaussian approximation is also presented. This last solution provides a trade-off between accuracy and time.

\section{The quotient ensemble eigenvalues distribution}

In the previous section, we showed that the PDF and CDF of mutual information depends on the JPDF $P\left(\lambda_{1}, \ldots, \lambda_{n}\right)$ of $\mathbf{W}$. In this section, we invoke the recently derived JPDF $P\left(\lambda_{1}, \ldots, \lambda_{n}\right)$ for a quotient comprising Wishart matrices [6]. We link this result to the $r$-point correlation function. Both the JPDF $P\left(\lambda_{1}, \ldots, \lambda_{n}\right)$ of $\mathbf{W}$ and the $r$-point correlation function will be used in the following sections to derive our proposed expressions.

Consider the quotient ensemble of random matrices $\mathbf{W}$ as defined in (7). The probability density of these $n \times n$ dimensional complex matrices was recently derived in [6]:

$$
\begin{aligned}
p_{W}(\mathbf{W}) \propto & e^{-a^{-1} \operatorname{tr} \mathbf{W}} \operatorname{det}(\mathbf{W})^{n_{A}-n} \\
& \times \Psi\left(n_{B}, n_{A}+n_{B}+n ;\left(b^{-1} \mathbf{I}_{n}+a^{-1} \mathbf{W}\right)\right) .
\end{aligned}
$$

Here, $\Psi(\cdot)$ is the confluent hypergeometric function of the second kind (Tricomi function) with matrix argument [30]:

$$
\begin{aligned}
\Psi(\alpha, \gamma ; \mathbf{X}) & =\frac{1}{\pi^{n(n-1) / 2} \prod_{j=1}^{n} \Gamma(\alpha-j+1)} \\
& \int_{\mathbf{Y}>0} e^{-\operatorname{tr}(\mathbf{X Y})}|\mathbf{Y}|^{\alpha-n}|\mathbf{I}+\mathbf{Y}|^{\gamma-\alpha-n} d \mathbf{Y},
\end{aligned}
$$

with $\operatorname{Re}(\mathbf{X})>0, \operatorname{Re}(\alpha)>(n-1)$ for convergence, and $\operatorname{Re}(\cdot)$ denotes the real part. It should be noted that the integral in (13) is over the set of positive definite matrices $(\mathbf{Y}>0)$.

The JPDF $P\left(\lambda_{1}, \ldots, \lambda_{n}\right)$ of eigenvalues of $\mathbf{W}$ exhibits a biorthogonal structure of Borodin type [31] and is given by [6]

$P\left(\lambda_{1}, \ldots, \lambda_{n}\right)=C_{n} \Delta_{n}(\{\lambda\}) \prod_{i=1}^{n} e^{-\lambda_{i} / a} \lambda_{i}^{n_{A}-n} \operatorname{det}\left[f_{j}\left(\lambda_{k}\right)\right]_{j, k=1, \ldots, n}$,

where

$$
\Delta_{n}(\{\lambda\})=\operatorname{det}\left[\lambda_{k}^{j-1}\right]_{j, k=1, \ldots, n}=\prod_{j>k}\left(\lambda_{j}-\lambda_{k}\right),
$$

is the Vandermonde determinant, and

$$
f_{j}\left(\lambda_{k}\right)=U\left(n_{B}-j+1, n_{A}+n_{B}-j+2 ; \frac{1}{b}+\frac{\lambda_{k}}{a}\right)
$$

is in terms of the usual confluent hypergeometric function $U(\cdot)$ of the second type (Tricomi function). ${ }^{1}$ 
The normalization factor in (14), $C_{n}$, turns out to be

$$
\begin{aligned}
C_{n}^{-1} & =n ! \operatorname{det}\left[h_{j, k}\right]_{j, k=1, \ldots, n} \\
& =n ! a^{n n_{A}-n(n-1) / 2} b^{n n_{B}} \prod_{j=1}^{n} \Gamma(j) \Gamma\left(n_{A}-j+1\right),
\end{aligned}
$$

with

$$
\begin{aligned}
h_{j, k} & =\int_{0}^{\infty} e^{-\lambda / a} \lambda^{n_{A}-n+k-1} f_{j}(\lambda) d \lambda \\
& =a^{n_{A}-n+k} \Gamma\left(n_{A}-n+k\right) U\left(n_{B}-j+1, n_{B}+n-j-k+2 ; \frac{1}{b}\right) .
\end{aligned}
$$

The $r$-point correlation function [32], $(1 \leq r \leq n)$, corresponding to (14) is given by [6]:

$$
\begin{aligned}
& R_{r}\left(\lambda_{1}, \ldots, \lambda_{r}\right)=\frac{n !}{(n-r) !} \int_{0}^{\infty} \cdots \int_{0}^{\infty} P\left(\lambda_{1}, \ldots, \lambda_{n}\right) d \lambda_{n} \cdots d \lambda_{r+1} \\
& =(-1)^{r} n ! C_{n} \prod_{l=1}^{r} e^{-\lambda_{l} / a} \lambda_{l}^{n_{A}-n} \operatorname{det}\left[\begin{array}{cc}
0 & {\left[\lambda_{j}^{k-1}\right]_{j=1, \ldots, r}} \\
{\left[f_{j}\left(\lambda_{k}\right)\right]_{j=1, \ldots, n}} & {\left[h_{j, k}\right] \begin{array}{c}
k=1, \ldots, n \\
k=1, \ldots, r \\
k=1, \ldots, n
\end{array}}
\end{array}\right] \text {, }
\end{aligned}
$$

where $\mathbb{Q}$ represents a $r \times r$ block with all entries zero.

The one-point function $R_{1}\left(\lambda_{1}\right)$ and the two-point function $R_{2}\left(\lambda_{1}, \lambda_{2}\right)$ will be useful in order to obtain the Gaussian approximation. We note that the one-point function is related to the marginal density as $p_{1}(\lambda)=R_{1}(\lambda) / n$, while the two-point function gives the JPDF of two eigenvalues as $p_{2}\left(\lambda_{1}, \lambda_{2}\right)=R_{2}\left(\lambda_{1}, \lambda_{2}\right) /(n(n-1))$.

\section{Extreme eigenvalues statistics}

Along with the mutual information PDF and CDF that depends on the distribution of all $n$ eigenvalues ${ }^{2}$ as shown in (10) and (11), respectively, the distribution of the extreme eigenvalues (the smallest/the largest) [33] also serve as important metric for analyzing the performance of MIMO systems [34-36].

In this section, we derive exact results for the distributions and densities of both the smallest eigenvalue $\left(\lambda_{\min }\right)$ and the largest eigenvalue $\left(\lambda_{\max }\right)$ of the quotient ensemble defined in (7). These are based on the general results summarized in [37]. We first present exact results for the gap probability which refers to the probability of finding no eigenvalue in a given interval. These are then used to obtain the densities of $\lambda_{\min }$ and $\lambda_{\max }$.

The probability that there are no eigenvalues between 0 and $x$, or equivalently the probability that all eigenvalues are greater than or equal to $x$ is given by

$$
E((0, x))=\int_{x}^{\infty} \ldots \int_{x}^{\infty} P\left(\lambda_{1}, \ldots, \lambda_{n}\right) d \lambda_{1} \ldots d \lambda_{n} .
$$

On the other hand, we have

$$
E((x, \infty))=\int_{0}^{x} \ldots \int_{0}^{x} P\left(\lambda_{1}, \ldots, \lambda_{n}\right) d \lambda_{1} \ldots d \lambda_{n},
$$

which gives the probability that there is no eigenvalue between $x$ and $\infty$, or equivalently that all eigenvalues are less than or equal to $x$. Inserting the JPDF given in (14) in the above equation and implementing Andréief's integration formula [38], at once, yield the result for the above gap probabilities in the present case. We have

$$
E((0, x))=n ! C_{n} \operatorname{det}\left[\chi_{j, k}((0, x))\right]_{j, k=1, \ldots, n},
$$

with the kernel $\chi_{j, k}((0, x))$ given by

$$
\begin{aligned}
\chi_{j, k}((0, x)) & =\int_{x}^{\infty} e^{-\lambda / a} \lambda^{n_{A}-n+k-1} f_{j}(\lambda) d \lambda \\
& =e^{-x / a} \sum_{r=0}^{n_{A}-n+k-1} \frac{\Gamma\left(n_{A}-n+k\right)}{\Gamma\left(n_{A}-n+k-r\right)} a^{r+1} x^{n_{A}-n+k-r-1} \\
& \times U\left(n_{B}-j+1, n_{A}+n_{B}-j-r+1 ; \frac{1}{b}+\frac{x}{a}\right)
\end{aligned}
$$

To obtain the finite-sum result in the second line above, we used the transformation $\mu=\lambda-x$, applied the binomial expansion on the resulting factor $(\mu+x)^{n_{A}-n+k-1}$, and finally performed term by term integration over $\mu$. We note that for $x \rightarrow 0, \chi_{j, k}((0, x))$ reduces to $h_{j, k}$ as in (18). In a similar way, we obtain

$$
E((x, \infty))=n ! C_{n} \operatorname{det}\left[\chi_{j, k}((x, \infty)]_{j, k=1, \ldots, n},\right.
$$

where $\chi_{j, k}((x, \infty)$ is given by

$$
\chi_{j, k}((x, \infty))=\int_{0}^{x} e^{-\lambda / a} \lambda^{n_{A}-n+k-1} f_{j}(\lambda) d \lambda=h_{j, k}-\chi_{j, k}((0, x)) .
$$

Now, since $E((0, x))$ gives the survival function ${ }^{3}(\mathrm{SF})$ or reliability function of the $\lambda_{\min }$, it can be used to obtain the corresponding PDF. It is given by

$p_{\lambda_{\min }}(x)=-\frac{d}{d x} E((0, x))=n ! C_{n} \sum_{\mu=1}^{n} \operatorname{det}\left[\phi_{j, k}^{(\mu)}(x)\right]_{j, k=1, \ldots, n}$,

where

$$
\phi_{j, k}^{(\mu)}(x)= \begin{cases}e^{-x / a} x^{n_{A}-n+k-1} f_{j}(x), & j=\mu, \\ \chi_{j, k}((0, x)), & j \neq \mu .\end{cases}
$$

Similarly, $E((x, \infty))$ is the CDF of the $\lambda_{\max }$, and hence the PDF of $\lambda_{\max }$ is obtained as

$p_{\lambda_{\max }}(x)=\frac{d}{d x} E((x, \infty))=n ! C_{n} \sum_{\mu=1}^{n} \operatorname{det}\left[\psi_{j, k}^{(\mu)}(x)\right]_{j, k=1, \ldots, n}$, 
with

$$
\psi_{j, k}^{(\mu)}(x)= \begin{cases}e^{-x / a} x^{n_{A}-n+k-1} f_{j}(x), & j=\mu, \\ \chi_{j, k}((x, \infty)), & j \neq \mu .\end{cases}
$$

We show in Section 6 that the above exact expressions agree perfectly with the Monte Carlo simulations.

\section{Proposed mutual information exact density and outage probability}

In this section, we present two exact ways to obtain the PDF and outage probability of mutual information. Moreover, the Gaussian approximation is also presented, since it leads to reasonable results and is more straightforward than the exact solutions.

\subsection{Exact results based directly on JPDF}

We first calculate the PDF of mutual information. For this, we notice that one of the integrals in (11) can be easily performed because of the Dirac-delta function. To see this, we write (11) as

$$
\begin{aligned}
p\left(\mathcal{I}_{A}\right)=\int_{0}^{\infty} \cdots \int_{0}^{\infty} \delta & \left(\log _{2} \frac{2^{\mathcal{I}_{A}}}{\prod_{j=1}^{n}\left(1+\lambda_{j}\right)}\right) \\
& \times P\left(\lambda_{1}, \ldots, \lambda_{n}\right) d \lambda_{1} \cdots d \lambda_{n} \\
=\ln 2 \int_{0}^{\infty} \cdots \int_{0}^{\infty} \delta & \left(\lambda_{1}+1-\frac{2^{\mathcal{I}_{A}}}{\prod_{j=2}^{n}\left(1+\lambda_{j}\right)}\right) \\
& \times \frac{2^{\mathcal{I}_{A}}}{\prod_{j=2}^{n}\left(1+\lambda_{j}\right)} P\left(\lambda_{1}, \ldots, \lambda_{n}\right) d \lambda_{1} \cdots d \lambda_{n} .
\end{aligned}
$$

In the second line, we used the property $\delta(F(z))=\delta(z-$ $\left.z_{0}\right) /\left|F^{\prime}\left(z_{0}\right)\right|$ for $z=\lambda_{1}$, with $z_{0}$ being the root of $F(z)=$ 0 . The $\lambda_{1}$ integral can now be performed using the result $\int_{0}^{\infty} \delta\left(z-z_{0}\right) G(z) d z=G\left(z_{0}\right) \Theta\left(z_{0}\right)$ to yield

$$
\begin{aligned}
p\left(\mathcal{I}_{A}\right)= & \ln 2 \int_{0}^{\infty} \cdots \int_{0}^{\infty} \frac{2^{\mathcal{I}_{A}}}{\prod_{j=2}^{n}\left(1+\lambda_{j}\right)} P\left(\frac{2^{\mathcal{I}_{A}}}{\prod_{j=2}^{n}\left(1+\lambda_{j}\right)}-1, \lambda_{2}, \ldots, \lambda_{n}\right) \\
& \times \Theta\left(2^{\mathcal{I}_{A}}-\prod_{j=2}^{n}\left(1+\lambda_{j}\right)\right) d \lambda_{n} \cdots d \lambda_{2} \\
= & \ln 2 \int_{0}^{u_{2}} \cdots \int_{0}^{u_{n}} \frac{2^{\mathcal{I}_{A}}}{\prod_{j=2}^{n}\left(1+\lambda_{j}\right)} P\left(\frac{2^{\mathcal{I}_{A}}}{\prod_{j=2}^{n}\left(1+\lambda_{j}\right)}-1, \lambda_{2}, \ldots, \lambda_{n}\right) d \lambda_{n} \cdots d \lambda_{2},
\end{aligned}
$$

where

$$
u_{\mu}=\frac{2^{\mathcal{I}_{A}}\left(1+\lambda_{2}\right)}{\prod_{j=2}^{\mu}\left(1+\lambda_{j}\right)}-1=\frac{2^{\mathcal{I}_{A}}}{\prod_{j=2}^{\mu-1}\left(1+\lambda_{j}\right)}-1 .
$$

It is to be noted that $u_{2}=2^{\mathcal{I}_{A}}-1$. In the second line of (30), we adjusted the integration limits of the variables in a particular way to take care of the theta function constraint in the first line. For instance, to take care of $\Theta\left(\alpha-z_{1} z_{2}\right)$, we can consider $z_{2}<\alpha / z_{1}$ for $z_{1}, z_{2}, \alpha>0$.
Special case $(n=2)$ : We have ([39], Eq. 6.40)

$$
p\left(\mathcal{I}_{A}\right)=\ln 2 \int_{0}^{2^{\mathcal{I}_{A}-1}} \frac{2^{\mathcal{I}_{A}}}{1+\lambda_{2}} P\left(\frac{2^{\mathcal{I}_{A}}}{1+\lambda_{2}}-1, \lambda_{2}\right) d \lambda_{2} .
$$

The outage probability can be written using (10) as

$$
p_{\text {out }}(R)=\int_{0}^{v_{1}} \cdots \int_{0}^{v_{n}} P\left(\lambda_{1}, \lambda_{2}, \ldots, \lambda_{n}\right) d \lambda_{n} \cdots d \lambda_{1},
$$

where

$$
v_{\mu}=\frac{2^{R}\left(1+\lambda_{1}\right)}{\prod_{j=1}^{\mu}\left(1+\lambda_{j}\right)}-1=\frac{2^{R}}{\prod_{j=1}^{\mu-1}\left(1+\lambda_{j}\right)}-1 .
$$

In (32), we again adopted the strategy of modifying the integration limits to take care of the theta function in (10). Another possible expression for the outage probability, using (30), can be written as

$$
\begin{gathered}
p_{\text {out }}(R)=\int_{0}^{R} p\left(\mathcal{I}_{A}\right) d \mathcal{I}_{A} \\
=\ln 2 \int_{0}^{R} \int_{0}^{u_{2}} \cdots \int_{0}^{u_{n}} \frac{2^{\mathcal{I}_{A}}}{\prod_{j=2}^{n}\left(1+\lambda_{j}\right)} P\left(\frac{2^{\mathcal{I}_{A}}}{\prod_{j=2}^{n}\left(1+\lambda_{j}\right)}-1, \lambda_{2}, \ldots, \lambda_{n}\right) \\
\times d \lambda_{n} \cdots d \lambda_{2} d \mathcal{I}_{A} .
\end{gathered}
$$

5.2 Exact results based on Laplace transform approach The Laplace transform of $p\left(\mathcal{I}_{A}\right)$ defined in (11) is given by

$$
\begin{aligned}
\tilde{p}(s) & =\mathcal{L}\left[p\left(\mathcal{I}_{A}\right)\right](s)=\int_{0}^{\infty} \cdots \int_{0}^{\infty} \\
& e^{-s \sum_{j=1}^{n} \log _{2}\left(1+\lambda_{j}\right)} P\left(\lambda_{1}, \ldots, \lambda_{n}\right) d \lambda_{1} \cdots d \lambda_{n} .
\end{aligned}
$$

We note that

$$
\tilde{p}(s)=\int_{0}^{\infty} e^{-s \mathcal{I}_{A}} p\left(\mathcal{I}_{A}\right) d \mathcal{I}_{A}=\sum_{k=0}^{\infty} \frac{(-1)^{k} s^{k}}{k !} \mathbb{E}\left[\mathcal{I}_{A}^{k}\right] .
$$

Thus, we see that the Laplace transform serves as the moment-generating function (MGF) for $\mathcal{I}_{A}$, since the moments of $\mathcal{I}_{A}$ can be obtained using the coefficients of powers of $s$ in the series expansion of $\tilde{p}(s)$. Using the JPDF given in (14), we obtain

$$
\begin{aligned}
\tilde{p}(s)=C_{n} \int_{0}^{\infty} & \cdots \int_{0}^{\infty} \Delta(\{\lambda\}) \prod_{l=1}^{n} e^{-\lambda_{l} / a} \lambda_{l}^{n_{A}-n}\left(1+\lambda_{l}\right)^{-(s / \ln 2)} d \lambda_{1} \cdots d \lambda_{n} \\
& \times \operatorname{det}\left[U\left(n_{B}-j+1, n_{A}+n_{B}-j+2 ; \frac{1}{b}+\frac{\lambda_{k}}{a}\right)\right]_{j, k=1, \ldots, n},
\end{aligned}
$$

where we used $\log _{2} z=\ln z / \ln 2$.

With the aid of Andréief's integration formula [38], the above result can be immediately cast in the form of a determinant 


$$
\tilde{p}(s)=n ! C_{n} \operatorname{det}\left[\eta_{j, k}(s)\right]_{j, k=1, \ldots, n},
$$

where

$$
\begin{aligned}
\eta_{j, k}(s)=\int_{0}^{\infty} & (1+\lambda)^{-(s / \ln 2)} \lambda^{n_{A}-n+k-1} e^{-\lambda / a} \\
& \times U\left(n_{B}-j+1, n_{A}+n_{B}-j+2 ; \frac{1}{b}+\frac{\lambda}{a}\right) d \lambda .
\end{aligned}
$$

The density of $\mathcal{I}_{A}$ follows by taking the inverse Laplace of $\widetilde{p}(s)$,

$$
p\left(\mathcal{I}_{A}\right)=\mathcal{L}^{-1}\{\tilde{p}(s)\}\left(\mathcal{I}_{A}\right) .
$$

The outage probability follows by taking the inverse Laplace of $s^{-1} \widetilde{p}(s)$ [10],

$$
p_{\text {out }}(R)=\mathcal{L}^{-1}\left\{s^{-1} \widetilde{p}(s)\right\}(R) .
$$

With the above results, we may recover the densities by performing numerical inversion of Laplace transform as in [10]. However, we make further analytical progress below to obtain an alternative expression for the PDF of mutual information.

Special case $(n=1)$ : Let us consider the $n=1$ case. The density of mutual information can be obtained from (11) as

$$
\begin{aligned}
p\left(\mathcal{I}_{A}\right)=C_{1} \int_{0}^{\infty} & \delta\left(\mathcal{I}_{A}-\log _{2}(1+\lambda)\right) e^{-\lambda / a} \lambda^{n_{A}-1} \\
& \times U\left(n_{B}, n_{A}+n_{B}+1 ; \frac{1}{b}+\frac{\lambda}{a}\right) d \lambda .
\end{aligned}
$$

This one-dimensional integral can be readily performed because of the presence of Dirac-delta function and yields the exact result for $n=1$ as

$$
\begin{aligned}
p\left(\mathcal{I}_{A}\right)= & \frac{a^{-n_{A}} b^{-n_{B}}}{\Gamma\left(n_{A}\right)}(\ln 2) 2_{A}^{\mathcal{I}}\left(2_{A}^{\mathcal{I}}-1\right)^{n_{A}-1} \\
& \times \exp \left(-\frac{2_{A}^{\mathcal{I}}-1}{a}\right) U\left(n_{B}, n_{A}+n_{B}+1 ; \frac{1}{b}+\frac{2_{A}^{\mathcal{I}}-1}{a}\right) .
\end{aligned}
$$

Comparing (40) with (37) evaluated for $n=1$, we arrive at the following inverse Laplace transform identity:

$$
\begin{aligned}
\mathcal{L}^{-1}\left[\int_{0}^{\infty} \lambda^{\gamma} e^{-\lambda / a}(1+\lambda)^{-s / \ln 2} U\left(\alpha, \beta, \frac{1}{b}+\frac{\lambda}{a}\right) d \lambda\right](t)= \\
(\ln 2) 2^{t} \exp \left(-\frac{\left(2^{t}-1\right)}{a}\right)\left(2^{t}-1\right)^{\gamma} U\left(\alpha, \beta, \frac{1}{b}+\frac{2^{t}-1}{a}\right) .
\end{aligned}
$$

With this interesting result in our hands, we can use the convolution property of the Laplace transform and write an expression for the PDF of mutual information for arbitrary $n$ as a $(n-1)$ fold integral. To this end, we expand the determinant in (37) and afterwards use the following result for inverse Laplace transform of product of $n$ functions, which follows from the result for product of two functions [40]

$$
\begin{aligned}
\mathcal{L}^{-1} & {\left[\widetilde{F}_{1}(s) \widetilde{F}_{2}(s) \cdots \widetilde{F}_{n}(s)\right]\left(x_{1}\right)=\int_{0}^{x_{1}} \int_{0}^{x_{2}} \cdots \int_{0}^{x_{n-1}} } \\
& F_{1}\left(x_{1}-x_{2}\right) F_{2}\left(x_{2}-x_{3}\right) \cdots F_{n-1}\left(x_{n-1}-x_{n}\right) F_{n}\left(x_{n}\right) d x_{n} \cdots d x_{3} d x_{2},
\end{aligned}
$$

where

$$
\mathcal{L}^{-1}\left[\widetilde{F}_{j}(s)\right](t)=F_{j}(t), \quad j=1, \ldots, n .
$$

Therefore, with the help of (41) in (38), we obtain the following expression:

$$
\begin{aligned}
p\left(\mathcal{I}_{A}\right)=n ! C_{n}(\ln 2)^{n} 2^{x_{1}} & \int_{0}^{x_{1}} \int_{0}^{x_{2}} \cdots \int_{0}^{x_{n-1}} \prod_{j>k}\left(2^{x_{j}-x_{j+1}}-2^{x_{k}-x_{k+1}}\right) \\
& \times \prod_{j=1}^{n}\left(2^{x_{j}-x_{j+1}}-1\right)^{n_{A}-n} \exp \left(-\frac{\left(2^{x_{j}-x_{j+1}}-1\right)}{a}\right) \\
& \times U\left(n_{B}-j+1, n_{A}+n_{B}-j+2 ; \frac{1}{b}+\frac{2^{x_{j}-x_{j+1}}-1}{a}\right) \\
& \times d x_{n} \cdots d x_{3} d x_{2},
\end{aligned}
$$

where $x_{1} \equiv \mathcal{I}_{A}$ and $x_{n+1} \equiv 0$.

Special case $(n=2)$ : We have the following explicit result

$$
\begin{aligned}
p\left(\mathcal{I}_{A}\right) & =\frac{a^{1-2 n_{A}} b^{-2 n_{B}}}{\Gamma\left(n_{A}\right) \Gamma\left(n_{A}-1\right)}(\ln 2)^{2} 2^{\mathcal{I}_{A}} \int_{0}^{\mathcal{I}_{A}}\left(2^{x}-2^{\mathcal{I}_{A}-x}\right)\left(2^{\mathcal{I}_{A}-x}-1\right)^{n_{A}-2}\left(2^{x}-1\right)^{n_{A}-2} \\
& \times \exp \left(-\frac{\left(2^{\mathcal{I}_{A}-x}+2^{x}-2\right)}{a}\right) U\left(n_{B}, n_{A}+n_{B}+1 ; \frac{1}{b}+\frac{2^{\mathcal{I}_{A}-x}-1}{a}\right) \\
& \times U\left(n_{B}-1, n_{A}+n_{B} ; \frac{1}{b}+\frac{2^{x}-1}{a}\right) d x .
\end{aligned}
$$

The outage probability, which is the CDF of the mutual information, can be written as the integral of (42) from 0 to $R$ as

$$
\begin{aligned}
p_{\text {out }}(R)= & \int_{0}^{R} p\left(x_{1}\right) d x_{1} \\
= & n ! C_{n}(\ln 2)^{n} \int_{0}^{R} \int_{0}^{x_{1}} \int_{0}^{x_{2}} \cdots \int_{0}^{x_{n-1}} 2^{x_{1}} \prod_{j>k}\left(2^{x_{j}-x_{j+1}}-2^{x_{k}-x_{k+1}}\right) \\
\times & \prod_{j=1}^{n}\left(2^{x_{j}-x_{j+1}}-1\right)^{n_{A}-n} \exp \left(-\frac{\left(2^{x_{j}-x_{j+1}}-1\right)}{a}\right) \\
\times & U\left(n_{B}-j+1, n_{A}+n_{B}-j+2 ; \frac{1}{b}+\frac{2^{x_{j}-x_{j+1}}-1}{a}\right) \\
& \quad \times d x_{n} \cdots d x_{3} d x_{2} d x_{1} .
\end{aligned}
$$

We should remark at this point that for the evaluation of PDF and outage probability of $\mathcal{I}_{A}$, as far as number of integrals is concerned, we have not gained anything. However, the above exact expressions provide an alternative route to calculate these quantities compared to 
the expressions derived in the last subsection, where we adopted the strategy of integrating the JPDF directly.

\subsection{Gaussian approximation}

The expressions for PDF and CDF presented above use the JPDF of the eigenvalues or Laplace transform, which involves the calculation of multiple integrals. A more straightforward method is to use the Gaussian approximation that depends only on integrals involving up to two eigenvalue densities.

The PDF of mutual information can be approximated by a Gaussian distribution as [10]

$$
p\left(\mathcal{I}_{A}\right) \approx \frac{1}{\sqrt{2 \pi \sigma_{\mathcal{I}_{A}}^{2}}} \exp \left(-\frac{\left(\mathcal{I}_{A}-\mu_{\mathcal{I}_{A}}\right)^{2}}{2 \sigma_{\mathcal{I}_{A}}^{2}}\right) .
$$

Correspondingly, the outage probability is given by

$$
p_{\text {out }}(R) \approx \frac{1}{2} \operatorname{erfc}\left(\frac{\mu_{\mathcal{I}_{A}}-R}{\sqrt{2 \sigma_{\mathcal{I}_{A}}^{2}}}\right),
$$

where $\operatorname{erfc}(\cdot)$ represents the complementary error function.

We propose below two approaches to calculate the mean and variance of the mutual information $\mathcal{I}_{A}$.

\subsubsection{Laplace transform based}

We can obtain an arbitrary moments of the mutual information using the Laplace transform results (34) and (35) as

$$
\mathbb{E}\left[\mathcal{I}_{A}^{k}\right]=\left.(-1)^{k} \frac{d^{k} \widetilde{p}(s)}{d s^{k}}\right|_{s=0} ; k=0,1,2, \ldots .
$$

In particular, the first moment (mean) and the second moment are obtained by differentiating the determinantbased expression in (34), and then setting $s=0$. We find

$$
\begin{gathered}
\mu_{\mathcal{I}_{A}}=\mathbb{E}\left[\mathcal{I}_{A}\right]=-n ! C_{n} \sum_{\mu=1}^{n} \operatorname{det}\left[\eta_{j, k}^{(\mu)}\right]_{j, k=1, \ldots, n}, \quad \text { (48) } \\
\mathbb{E}\left[\mathcal{I}_{A}^{2}\right]=n ! C_{n}\left(\sum_{\mu=1}^{n} \operatorname{det}\left[\xi_{j, k}^{(\mu)}\right]_{j, k=1, \ldots, n}+2 \sum_{1 \leq \mu<\nu \leq n} \operatorname{det}\left[\xi_{j, k}^{(\mu, \nu)}\right]_{j, k=1, \ldots, n}\right) .
\end{gathered}
$$

Here,

$$
\eta_{j, k}^{(\mu)}= \begin{cases}-\int_{0}^{\infty} \log _{2}(1+\lambda) \lambda^{n_{A}-n+k-1} e^{-\lambda / a} f_{j}(\lambda) d \lambda & j=\mu, \\ h_{j, k} & j \neq \mu,\end{cases}
$$

$\xi_{j, k}^{(\mu)}= \begin{cases}\int_{0}^{\infty}\left(\log _{2}(1+\lambda)\right)^{2} \lambda^{n_{A}-n+k-1} e^{-\lambda / a} f_{j}(\lambda) d \lambda & j=\mu, \\ h_{j, k} & j \neq \mu,\end{cases}$ $\xi_{j, k}^{(\mu, v)}= \begin{cases}-\int_{0}^{\infty} \log _{2}(1+\lambda) \lambda^{n_{A}-n+k-1} e^{-\lambda / a} f_{j}(\lambda) d \lambda & j=\mu \text { or } v, \\ h_{j, k} & j \neq \mu .\end{cases}$

Here, $f_{j}(\lambda)$ and $h_{j, k}$ are as in (16) and (18), respectively. The variance of $\mathcal{I}_{A}$ can be calculated as $\sigma_{\mathcal{I}_{A}}^{2}=$ $\mathbb{E}\left[\mathcal{I}_{A}^{2}(\mathbf{W})\right]-\left(\mathbb{E}\left[\mathcal{I}_{A}(\mathbf{W})\right]\right)^{2}$. Using the mean and variance of the mutual information in (45) and (46), we obtain the approximations for the PDFs of the mutual information and outage probability, respectively.

\subsubsection{Correlation function based}

Under this approach, we use the correlation function expression (19) to obtain the mean and variance of the mutual information. The $\mu_{\mathcal{I}_{A}}$ can be obtained by averaging over the ensemble of $\mathbf{W}$ as [9]

$$
\begin{aligned}
\mu_{\mathcal{I}_{A}} & =\mathbb{E}\left[\mathcal{I}_{A}(\mathbf{W})\right] \\
& =\mathbb{E}\left[\sum_{j=0}^{n} \log _{2}\left(1+\lambda_{j}\right)\right] \\
& =n \mathbb{E}\left[\log _{2}\left(1+\lambda_{1}\right)\right],
\end{aligned}
$$

and using the one-point function $R_{1}\left(\lambda_{1}\right)$, this can be written as

$$
\mu_{\mathcal{I}_{A}}=\int_{0}^{\infty} R_{1}\left(\lambda_{1}\right) \log _{2}\left(1+\lambda_{1}\right) d \lambda_{1} .
$$

Similarly, the $\sigma_{\mathcal{I}_{A}}^{2}$ can be obtained as [10]

$$
\begin{aligned}
\sigma_{\mathcal{I}_{A}}^{2} & =\mathbb{E}\left[\mathcal{I}_{A}^{2}(\mathbf{W})\right]-\left(\mathbb{E}\left[\mathcal{I}_{A}(\mathbf{W})\right]\right)^{2} \\
& =\mathbb{E}\left[\left(\sum_{j=1}^{n} \log _{2}\left(1+\lambda_{j}\right)\right)^{2}\right]-\mu_{\mathcal{I}_{A}}^{2} \\
& =\mathbb{E}\left[\sum_{j, k=1}^{n} \log _{2}\left(1+\lambda_{j}\right) \log _{2}\left(1+\lambda_{k}\right)\right]-\mu_{\mathcal{I}_{A}}^{2} \\
& =\mathbb{E}\left[\sum_{j=1}^{n} \log _{2}^{2}\left(1+\lambda_{j}\right)+\sum_{j \neq k} \log _{2}\left(1+\lambda_{j}\right) \log _{2}\left(1+\lambda_{k}\right)\right]-\mu_{\mathcal{I}_{A}}^{2} \\
& =n \mathbb{E}\left[\log _{2}^{2}\left(1+\lambda_{1}\right)\right]+n(n-1) \mathbb{E}\left[\log _{2}\left(1+\lambda_{1}\right) \log _{2}\left(1+\lambda_{2}\right)\right]-\mu_{\mathcal{I}_{A}}^{2} .
\end{aligned}
$$

Therefore, with the aid of one-point and two-point correlation functions (19), this can be written as

$$
\begin{aligned}
\sigma_{\mathcal{I}_{A}}^{2} & =\int_{0}^{\infty} R_{1}\left(\lambda_{1}\right) \log _{2}^{2}\left(1+\lambda_{1}\right) d \lambda_{1} \\
& +\int_{0}^{\infty} \int_{0}^{\infty} R_{2}\left(\lambda_{1}, \lambda_{2}\right) \log _{2}\left(1+\lambda_{1}\right) \log _{2}\left(1+\lambda_{2}\right) d \lambda_{1} d \lambda_{2}-\mu_{\mathcal{I}_{A}}^{2} .
\end{aligned}
$$

We note that while obtaining the exact density requires evaluation of $n-1$ or $n$-fold integrals, for Gaussian 
approximation, we need to perform up to a twofold integral. Both the approaches mentioned above can be easily implemented in symbolic manipulation computational packages, such as Mathematica. The first approach based on the Laplace transform is preferable, as it gives the result in terms of determinants where only some of the elements involve onefold integrals. The second approach, on the other hand, involves up to twofold integrals of determinantal expressions.

\section{Numerical results}

In this section, we present a numerical example in order to validate the exact expressions proposed in this work. We begin with the comparison of results concerning the eigenvalues of the quotient ensemble, viz., the marginal density, and probability distributions and densities of the extreme eigenvalues. Afterwards, we move over to examine the behavior of the mutual information. As will be shown, there is a perfect match between the results from Monte Carlo simulations and the exact results presented in the preceding sections.

We consider the following scenario. Suppose that user A transmits with $a=1\left(\mathrm{SNR}_{A}=n_{A} \times a=4 \times 1=6.02\right.$ $\mathrm{dB})$, and user B transmits with $b=1 / 3\left(\mathrm{SNR}_{B}=n_{B} \times b=\right.$ $5 \times 1 / 3=2.21 \mathrm{~dB}$ ). In Fig. 2 , we show the marginal density of eigenvalues for $n=3$, while in Figs. 3 and 4 we display the probability distributions and densities of the extreme eigenvalues.

Figure 5 shows the PDF of mutual information for $n=$ $\{2,3,4\}$. Notice that as the number of receiving antennas increases, the $\mu_{\mathcal{I}_{A}}$ also increases. When we double the number of antennas from $n=2$ to $n=4$, the $\mu_{\mathcal{I}_{A}}$ goes from 2.56 to 4.93, almost a twofold increase. This result is in accordance with the well-known result that the slope of the curve increases with $\min \left(n, n_{A}\right)[4]$. Note also that the distributions possess Gaussian-like shapes. For comparison, we have plotted the Gaussian approximation using $\mu_{\mathcal{I}_{A}}$ and $\sigma_{\mathcal{I}_{A}}^{2}$.

The outage probability is shown in Fig. 6. Again, by increasing the number of receiving antennas, the outage probability decreases. For example, for a rate of $3 \mathrm{bits} / \mathrm{s} / \mathrm{Hz}$, the outage probability is $\approx 90 \%$ with $n=2$ antennas and goes down to less than $1 \%$ for $n=4$ antennas. An outage probability of $1 \%$ allows a bit rate of 1.2 and $2.1 \mathrm{bits} / \mathrm{s} / \mathrm{Hz}$ with $n=2$ and $n=3$ antennas, respectively. Notice that the Gaussian approximation is indistinguishable for outage probabilities above $10 \%$ for any $n$. For outage probability of $1 \%$, the error by using this approximation is less than 0.2 bits/s/Hz.

We show the outage rate as function of $a$ and $n$ in Fig. 7 for $1 \%$ of outage probability with $0 \leq a \leq 30 \mathrm{~dB}, n=$ $\{2,3,4\}, n_{A}=4, n_{B}=5, b=1$. Note that the increase in outage rate is close to linear with $a$. We used only Gaussian approximation to show that, for this purpose, this simpler method presents good results.

\section{Conclusions}

We considered the quotient ensemble involving two Wishart matrices. We worked out exact closed-form expressions for the probability distributions and densities of the extreme eigenvalues. Afterwards, we derived exact expressions for the probability density and outage probability of mutual information of a two-user MIMO MAC network over block Rayleigh fading channels. These expressions allow the analytical evaluation of the probability density and outage beside the current numerical evaluation methods such as Monte Carlo. The exact expressions are presented in two different ways, Laplace transform and by direct integration of joint probability

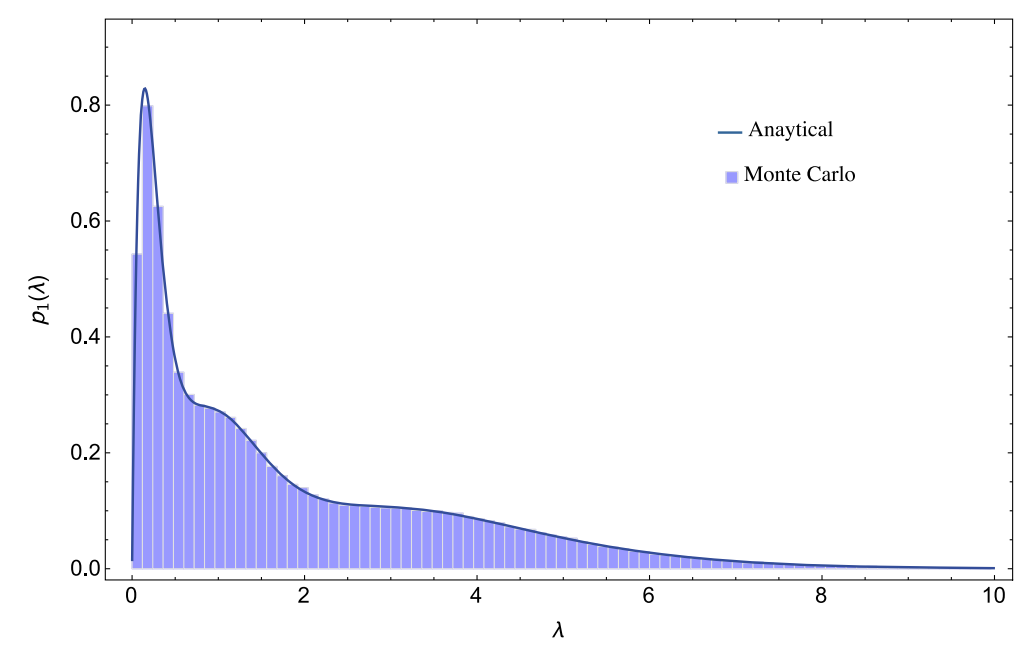

Fig. 2 Marginal density of eigenvalues of the quotient ensemble. The parameters used are $n=3, n_{A}=4, n_{B}=5, a=1$, and $b=1 / 3$ 


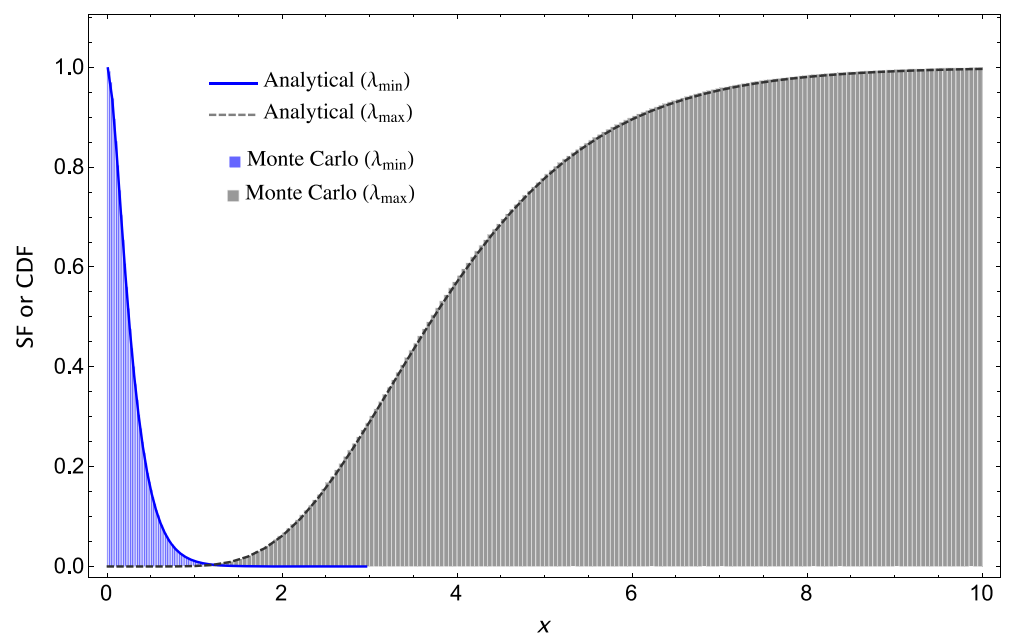

Fig. $3 \mathrm{SF}$ of the smallest eigenvalue $\left(\lambda_{\min }\right)$ and CDF of the largest eigenvalue ( $\left.\lambda_{\max }\right)$, as given by (22) and (24). The parameter values are $n=3$, $n_{A}=4, n_{B}=5$, and $b=1 / 3$

density function of eigenvalues coming from the quotient ensemble.

We showed that the density of mutual information exhibits a Gaussian-like shape. Therefore, besides the exact expressions, we derived expressions to evaluate the mean and variance to invoke the Gaussian approximation method. This approximation method offers a trade-off between complexity and accuracy. For outage probability, the Gaussian approximation shows excellent match with the exact results for outages above 10\%. For lower values, the Gaussian approximation error is relatively small so that we consider the method acceptable due its simplicity of implementation.
Finally, as an example of an application of the derived expressions, we evaluated the effect of the number of receiver antennas in the distribution and outage probability of the receiver. We noted a twofold increase in the mean value of mutual information when we double the number of receiving antennas. On the other hand, the outage rate increased about three times in the low signal to noise ratio regime.

\section{Endnotes}

${ }^{1}$ To avoid any confusion, we have used distinct symbols to represent confluent hypergeometric function with matrix argument $(\Psi)$ and that with scalar argument $(U)$.

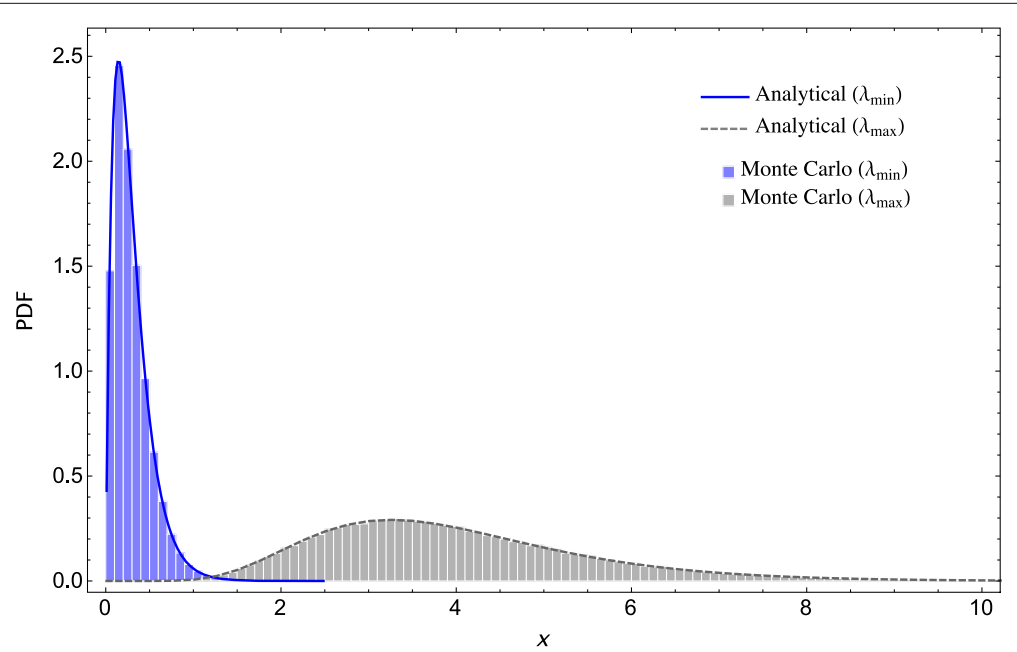

Fig. 4 PDF of the smallest eigenvalue $\left(\lambda_{\min }\right)$ and that of the largest eigenvalue $\left(\lambda_{\max }\right)$, as given by (26) and (28). The parameter values are $n=3$, $n_{A}=4, n_{B}=5$, and $b=1 / 3$ 


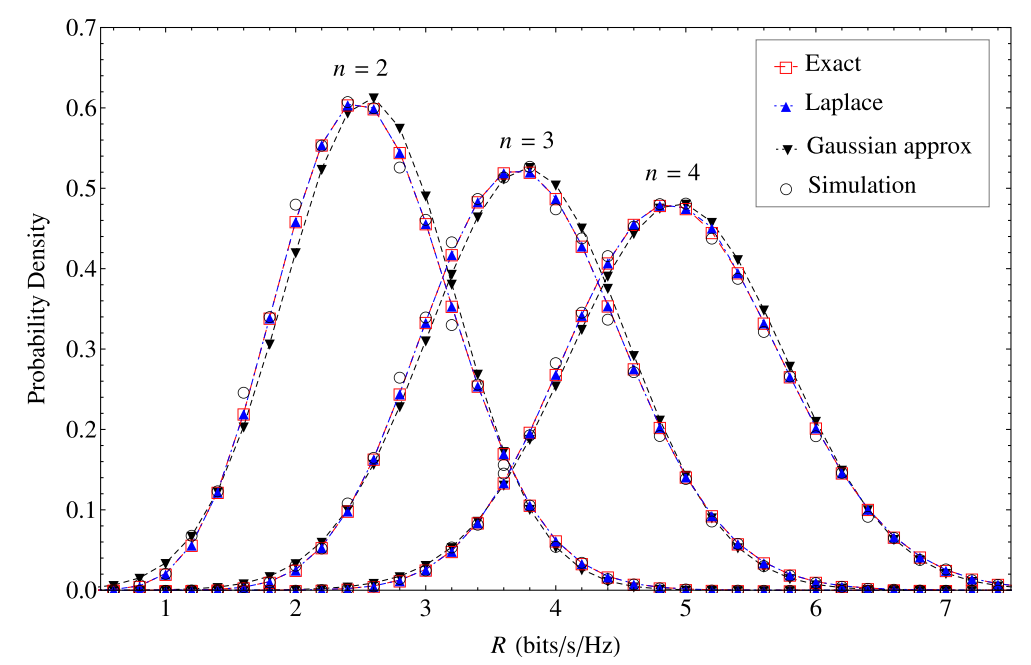

Fig. 5 Mutual information probability density for $n=\{2,3,4\}, n_{A}=4, n_{B}=5, a=1$, and $b=1 / 3$

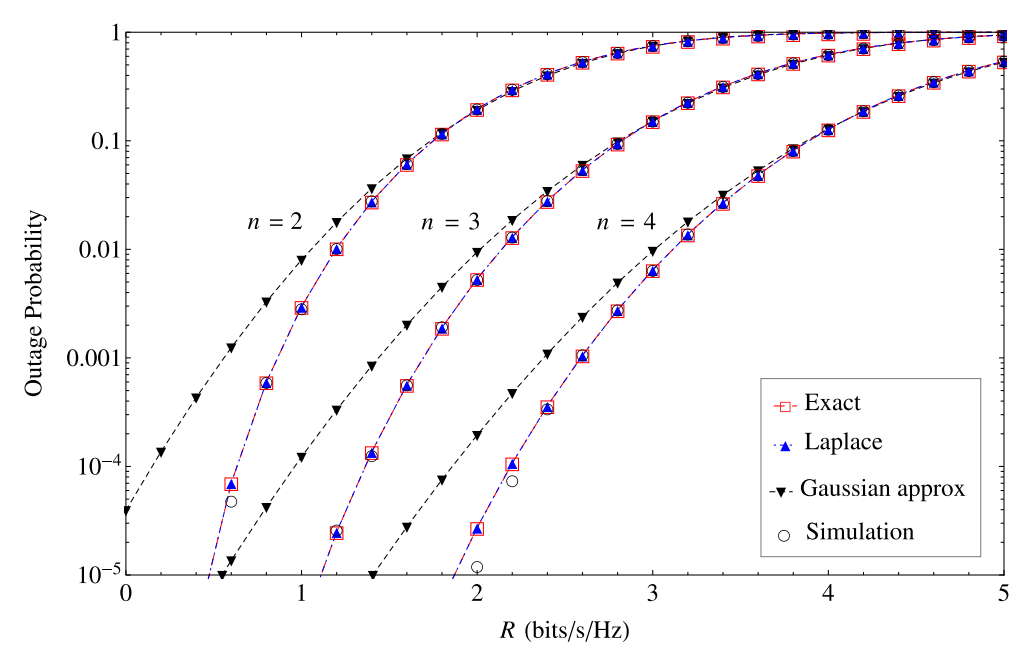

Fig. 6 Mutual information outage probability for $n=\{2,3,4\}, n_{A}=4, n_{B}=5, a=1$, and $b=1 / 3$

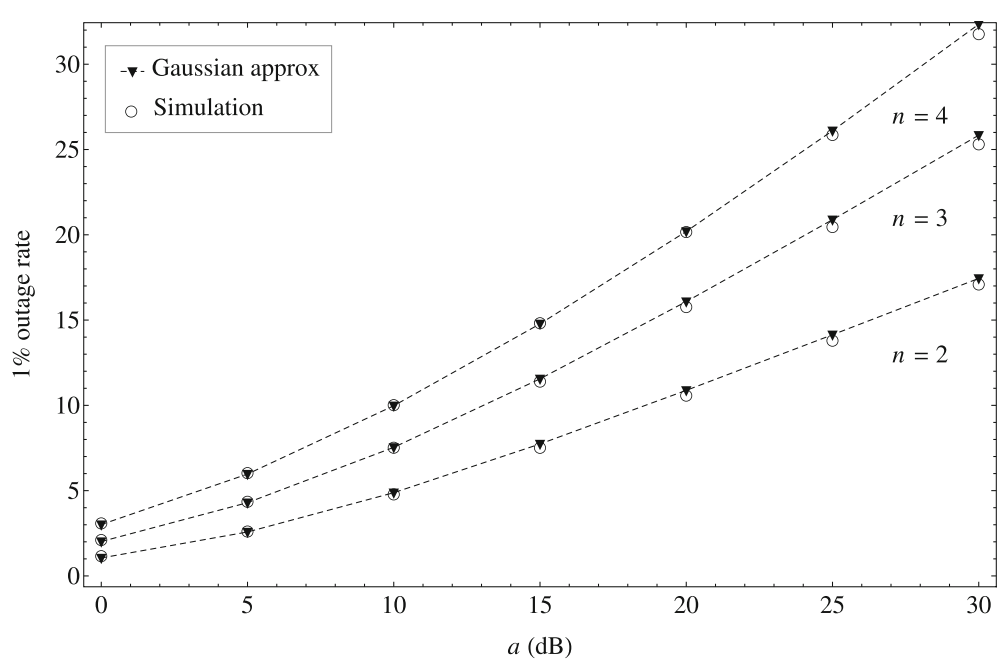

Fig. $71 \%$ outage rate for different $a$ and $n=\{2,3,4\}, n_{A}=4, n_{B}=5, b=1 / 3$ 


\section{${ }^{2}$ With exception for the Gaussian approximation case that will be shown in Section 5 . \\ ${ }^{3}$ Survival function and cumulative distribution function are related as $\mathrm{SF}=1-\mathrm{CDF}$.}

\author{
Acknowledgements \\ The authors would like to thank the anonymous reviewers for their careful \\ reading and insightful comments. The authors would like to thank the editor \\ and the reviewers for providing valuable comments that resulted in the \\ quality improvement of our work.
}

\section{Funding}

G. Pivaro was supported by CAPES-Brazil under Grant BEX10714/14-6. G. Fraidenraich was supported in part by Capes and CNPq, Brazil.

\section{Authors' contributions}

All authors contributed to the work. All authors read and approved the final manuscript.

\section{Publisher's Note}

Springer Nature remains neutral with regard to jurisdictional claims in published maps and institutional affiliations.

\section{Competing interests}

The authors declare that they have no competing interests.

\section{Author details}

${ }^{1}$ Department of Communications, State University of Campinas (Unicamp), Campinas, SP, Brazil. ²Department of Physics, Shiv Nadar University, Gautam Buddha Nagar, Uttar Pradesh, India.

Received: 22 September 2016 Accepted: 10 March 2017

Published online: 20 April 2017

\section{References}

1. A El Gamal, YH Kim, Network information theory. (Cambridge university press, Cambridge, 2011)

2. Z Ding, et al., in IEEE Communications Magazine, vol. 55, no. 2. Application of non-orthogonal multiple access in LTE and 5G networks, (2017), pp. 185-191

3. Q Sun, S Han, I Chin-Lin, Z Pan, On the Ergodic Capacity of Systems. IEEE Wirel. Commun. Lett. 4(4), 405-408 (2015)

4. D Tse, P Viswanath, Fundamentals of wireless communication. (Cambridge university press, Cambridge, 2005)

5. AT James, The distribution of the latent roots of the covariance matrix. Ann. Math. Stat. 31(1), 151-158 (1960)

6. S Kumar, Random matrix ensembles involving Gaussian Wigner and Wishart matrices, and biorthogonal structure. Phys. Rev. E. 92(3), 032903 (2015)

7. A Goldsmith, SA Jafar, N Jindal, S Vishwanath, Capacity limits of MIMO channels. IEEE J. Sel. Areas Commun. 21(5), 684-702 (2003)

8. Z Ding, R Schober, HV Poor, in 2016 IEEE International Conference on Communications (ICC). On the design of MIMO-NOMA downlink and uplink transmission (IEEE, Kuala Lumpur, 2016), pp. 1-6

9. E Telatar, Capacity of multi-antenna Gaussian channels. Eur. Trans Telecommun. 10(6), 585-595 (1999)

10. Z Wang, G Giannakis, Outage mutual information of space-time MIMO channels. IEEE Trans. Inf. Theory. 50(4), 657-662 (2004)

11. H Shin, JH Lee, in 2003 IEEE International Conference on Communications (ICC). Closed-form formulas for ergodic capacity of MIMO Rayleigh fading channels, vol. 5 (IEEE, Anchorage, 2003), pp. 2996-3000

12. P Smith, L Garth, Exact capacity distribution for dual MIMO systems in Ricean fading. IEEE Commun. Lett. 8(1), 18-20 (2004)

13. G Fraidenraich, $\mathrm{O}$ Leveque, J Cioffi, On the MIMO channel capacity for the dual and asymptotic cases over Hoyt channels. IEEE Commun. Lett. 11(1), 31-33 (2007)

14. S Kumar, A Pandey, Random matrix model for Nakagami-Hoyt fading. IEEE Trans. Inf. Theory. 56(5), 2360-2372 (2010)
15. G Taricco, Further results on the asymptotic mutual information of Rician fading MIMO channels. IEEE Trans. Inf. Theory. 59(2), 894-915 (2013)

16. S Li, M McKay, Y Chen, On the distribution of MIMO mutual information: an in-depth Painlev-based characterization. IEEE Trans. Inf. Theory. 59(9), 5271-5296 (2013)

17. S Ghosh, B Rao, J Zeidler, Outage-efficient strategies for multiuser MIMO networks with channel distribution information. IEEE Trans. Signal Process. 58(12), 6312-6324 (2010)

18. J Park, Y Sung, D Kim, H Poor, Outage probability and outage-based robus beamforming for MIMO interference channels with imperfect channel state information. IEEE Trans. Wirel. Commun. 11(10), 3561-3573 (2012)

19. RU Choi, M Ivrlac, R Murch, W Utschick, On strategies of multiuser MIMO transmit signal processing. IEEE Trans. Wirel. Commun. 3(6), 1936-1941 (2004)

20. R Hunger, M Joham, C Guthy, W Utschick, in 2009 Forty-Third Asilomar Conference on Signals, Systems and Computers. Weighted sum rate maximization in the MIMO MAC with linear transceivers: asymptotic results (IEEE, Pacific Grove, 2009), pp. 1528-1532

21. F Negro, S Shenoy, I Ghauri, D Slock, in Information Theory and Applications Workshop (ITA), 2010. On the MIMO interference channel (IEEE, San Diego, 2010), pp. 1-9

22. Z Ding, R Schober, HV Poor, A general MIMO framework for NOMA downlink and uplink transmission based on signal alignment. IEEE Trans. Wirel. Commun. 15(6), 4438-4454 (2016)

23. Z Ding, F Adachi, HV Poor, The application of MIMO to non-orthogonal multiple access. IEEE Trans. Wirel. Commun. 15(1), 537-552 (2016)

24. D Zhang, K Yu, Z Wen, T Sato, in 2016 IEEE 83rd Vehicular Technology Conference (VTC Spring). Outage probability analysis of NOMA within massive MIMO systems (IEEE, Nanjing, 2016), pp. 1-5

25. S Chen, K Peng, H Jin, J Song, Analysis of outage capacity of NOMA: SIC vs.JD. Tsinghua Sci. Technol. 21(5), 538-543 (2016)

26. B Wang, J Zhang, A Host-Madsen, On the capacity of MIMO relay channels. IEEE Trans. Inf. Theory. 51(1), 29-43 (2005)

27. G Pivaro, G Fraidenraich, C Ferreira Dias, Outage probability for MIMO relay channel. IEEE Trans. Commun. 62(11), 3791-3800 (2014)

28. S Kumar, GF Pivaro, G Fraidenraich, CF Dias, On the exact and approximate eigenvalue distribution for sum of Wishart matrices (2015) arXiv preprint arXiv:1504.00222

29. M Abramowitz, IA Stegun, Handbook of mathematical functions: with formulas, graphs, and mathematical tables. ser. Dover Books on Mathematics. (Dover Publications, Inc., New York, 2012)

30. RM Joshi, JMC Joshi, Confluent hypergeometric function of second kind with matrix argument. Indian J. Pure Appl. Math. 16, 627-636 (1985)

31. A Borodin, Biorthogonal ensembles. Nucl. Phys. B. 536(3), 704-732 (1998)

32. M Mehta, Random matrices, ser. Pure and Applied Mathematics. (Elsevier Inc., San Diego, 2004)

33. CG Khatri, Distribution of the largest or the smallest characteristic root under null hypothesis concerning complex multivariate normal populations. Ann. Math. Statist. 35(40), 1807-1810 (1964)

34. MS Alouini, Largest eigenvalue of complex Wishart matrices and performance analysis of MIMO MRC systems. IEEE J. Sel. Areas Commun. 21(3), 418-426 (2003)

35. A Zanella, M Chiani, in 2008 IEEE Global Telecommunications Conference. The PDF of the $l$ th largest eigenvalue of central Wishart matrices and its application to the performance analysis of MIMO systems (IEEE, New Orleans, 2008), pp. 1-6

36. A Zanella, M Chiani, MZ Win, in 2008 IEEE International Conference on Communications. A general framework for the distribution of the eigenvalues of Wishart matrices (IEEE, Beijing, 2008), pp. 1271-1276

37. S Kumar, Gap probabilities and densities of extreme eigenvalues of random matrices: Exact results, arXiv:1507.08830, Jul. 2015.

38. C Andréief, Note sur une relation les intégrales définies des produits des fonctions. Mém. De La Soc. Sci. Bordeaux. 2, 1-14 (1883)

39. A Papoulis, SU Pillai, Probability, random variables, and stochastic processes ser. McGraw-Hill series in electrical engineering: Communications and signal processing. (Tata McGraw-Hill, New York, 2002)

40. RN Bracewell, The Fourier transform and its applications, 3rd edn, (Mcgraw-Hill science/engineering/math, New York, 2000) 